\title{
A uniformly converging scheme for fractal conservation laws
}

Jérôme Droniou and Espen R. Jakobsen

\begin{abstract}
The fractal conservation law $\partial_{t} u+\partial_{x}(f(u))+(-\Delta)^{\alpha / 2} u=0$ changes characteristics as $\alpha \rightarrow 2$ from non-local and weakly diffusive to local and strongly diffusive. In this paper we present a corrected finite difference quadrature method for $(-\Delta)^{\alpha / 2}$ with $\alpha \in[0,2]$, combined with usual finite volume methods for the hyperbolic term, that automatically adjusts to this change and is uniformly convergent with respect to $\alpha \in[\eta, 2]$ for any $\eta>0$. We provide numerical results which illustrate this asymptotic-preserving property as well as the non-uniformity of previous finite difference or finite volume type of methods.
\end{abstract}

\section{Introduction}

We consider the following fractional conservation law

$$
\begin{array}{ll}
\partial_{t} u_{\alpha}+\partial_{x}\left(f\left(u_{\alpha}\right)\right)+\mathscr{L}_{\alpha}\left[u_{\alpha}\right]=0, & t>0, x \in \mathbb{R}, \\
u_{\alpha}(0, x)=u_{\text {ini }}(x), & x \in \mathbb{R},
\end{array}
$$

where $\alpha \in[0,2], \mathscr{L}_{\alpha}=(-\Delta)^{\alpha / 2}$,

$$
u_{\text {ini }} \in L^{\infty}(\mathbb{R}) \cap B V(\mathbb{R}) \quad \text { and } \quad f: \mathbb{R} \rightarrow \mathbb{R} \text { is locally Lipschitz-continuous. }
$$

Such models appear for example in mathematical finance, gas detonation or semiconductor growth $[23,26,11,1]$. The fractional Laplacian $\mathscr{L}_{\alpha}=(-\Delta)^{\alpha / 2}$ can be

Jérôme Droniou

School of Mathematical Sciences, Monash University, Victoria 3800, Australia, e-mail: jerome.droniou@monash.edu

Espen R. Jakobsen

Department of Mathematical Sciences, Norwegian University of Science and Technology, 7491

Trondheim, Norway, e-mail: erj@math.ntnu.no 
defined e.g. as a Fourier multiplier, but for our purpose the following equivalent definition, valid for any $\varphi \in C_{c}^{\infty}(\mathbb{R})$ (set of smooth compactly supported functions), is more useful:

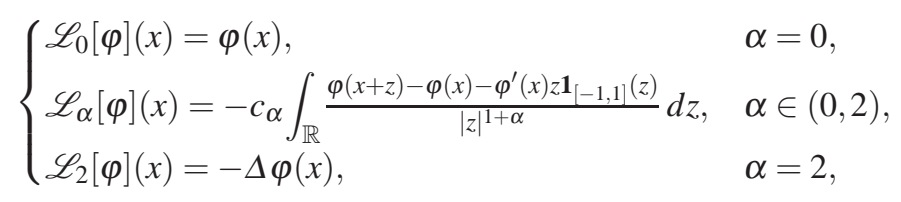

where $\mathbf{1}_{[-1,1]}$ is the characteristic function of $[-1,1], c_{\alpha}=(2 \pi)^{\alpha} \frac{\alpha \Gamma\left(\frac{1+\alpha}{2}\right)}{2 \pi^{\frac{1}{2}+\alpha} \Gamma\left(1-\frac{\alpha}{2}\right)}$ and $\Gamma$ is the Euler function [15].

As $\alpha \rightarrow 2$, the operator $\mathscr{L}_{\alpha}$ changes nature and properties. For $\alpha \in(0,2)$, $\mathscr{L}_{\alpha}$ is a non-local pseudo-differential operator of order $<2$, and it has relatively weak diffusive properties since the decay at infinity of the fundamental solution of $\partial_{t} u+\mathscr{L}_{\alpha}[u]=0$ is polynomial. At $\alpha=2, \mathscr{L}_{\alpha}=-\Delta$ is a local operator with strong diffusive properties and a fundamental solution with super-exponential decay. When $\alpha$ vary over [0,2], the qualitative behaviour of the solution $u_{\alpha}$ of (1) also changes. In the case that $\alpha=2$, it is well-known that $u_{\alpha}$ becomes instantly smooth for $t>0$ even when the initial data is discontinuous. On the contrary, for $\alpha=0$, the solution may develop shocks and uniqueness of the solution requires additional entropy conditions and the corresponding notion of entropy solution [22]. The study of the fractional case $\alpha \in(0,2)$ dates back to [6], with some restrictions on $\alpha$ and $f$. The first complete study in the case $\alpha>1$ for any locally Lipschitz $f$ and bounded initial data $u_{\text {ini }}$ can be found in [14]. Here it is proved that the solution becomes instantly smooth even if $u_{\text {ini }}$ is only bounded (see also [15]). If $\alpha<1$, then the solution can develop shocks [4] and the weak solution need not be unique [3]. The notion of entropy solution of [2] is therefore required to obtain a well-posed formulation.

There exists a vast literature on the numerical approximation of scalar conservation laws (i.e. (1) without $\mathscr{L}_{\alpha}$ ), see e.g. [17, 18, 19] and references therein. The study of numerical methods for fractal conservation laws is much more recent with a corresponding less extensive literature. Probabilistic methods have been studied in $[21,24]$, but must be applied to the equation satisfied by $\partial_{x} u_{\alpha}$ in order to avoid noisy results, and recovering from this a numerical approximation of $u_{\alpha}$ may be challenging in dimension greater than 1. Deterministic methods for (1) like finite difference, volume, and element methods (discontinuous Galerkin) are given in [13, 8, 10], while a high order spectral vanishing viscosity method is introduced in [9]. The latter method and its analysis is very different from the former three methods, with convergence and (non-optimal) error estimates that are independent of $\alpha \in(0,2)$. As opposed to the spectral method, the other methods are monotone or have low order monotone variants.

Surprisingly, for all the non-spectral monotone methods the convergence deteriorates as $\alpha \rightarrow 2$, and the schemes themselves are not even defined in the limit $\alpha=2$. The purpose of this paper is to present an asymptotic-preserving monotone scheme for (1) defined for any $\alpha \in[0,2]$, i.e. a scheme that provides a monotone 
approximation of $u_{\alpha}$ which is uniform with respect to $\alpha \in[0,2]$. In particular, our scheme naturally adapts to the change of behaviour of $\mathscr{L}_{\alpha}$ as $\alpha \rightarrow 2$ and $\alpha \rightarrow 0$ and its convergence properties do not deteriorate in these extreme cases. The idea behind our scheme is to add a correction term in the form of a suitably chosen vanishing local viscosity term. Similar ideas have been used for other equations before, see e.g. [12] for linear equations and [20] for fully nonlinear equations. A stochastic interpretation can be found in [5].

This paper is organised as follows. The numerical method is presented in Section 2 , and its asymptotic-preserving characteristics are discussed. Due to lack of space and the technical nature of the proofs, we skip them and refer instead to [16]. In Sections 3 and 4, we define precisely what asymptotic preserving means and the we give a couple numerical simulations to illustrate this property of the method.

\section{The scheme}

The new scheme is based on monotone convervative finite difference approximations of the local terms combined with quadrature, truncation of $\frac{1}{|z|^{1+\alpha}}$ near the singularity, and a second order correction term (vanishing viscosity) for the non-local term. Except for the correction term, the scheme is similar to the schemes of $[13,8]$ and of [10] with $P_{0}$-elements. It is monotone, conservative, and converges in $L_{l o c}^{1}$ uniformly in $\alpha \in[\eta, 2]$ for all $\eta>0$.

For given space and time steps $\delta x, \delta t>0$, we introduce the grid $t_{n}:=n \delta t$ and $x_{i}:=i \delta x+\frac{\delta x}{2}$ for $n \in \mathbb{N}_{0}$ and $i \in \mathbb{Z}$. We identify sequences $\left(\varphi_{i}\right)_{i \in \mathbb{Z}}$ of numbers with piecewise constant functions $\varphi_{\delta x}: \mathbb{R} \rightarrow \mathbb{R}$ equal to $\varphi_{i}$ on $[i \delta x,(i+1) \delta x)$ for all $i \in \mathbb{Z}$. Similarly, $\left(\varphi_{i}^{n}\right)_{n \geq 0, i \in \mathbb{Z}}$ is identified with $\varphi_{\delta x, \delta t}:[0, \infty) \times \mathbb{R} \rightarrow \mathbb{R}$ equal to $\varphi_{i}^{n}$ on $[n \delta t,(n+1) \delta t) \times[i \delta x,(i+1) \delta x)$ for all $n \geq 0$ and $i \in \mathbb{Z}$. The discretisation of (1) can then we written as: find $u_{\alpha, \delta x, \delta t}=\left(u_{i}^{n}\right)_{n \geq 0, i \in \mathbb{Z}}$ such that

$$
\begin{gathered}
u_{i}^{0}=\frac{1}{\delta x} \int_{[i \delta x,(i+1) \delta x)} u_{0}(x) d x \quad \text { for all } i \in \mathbb{Z}, \\
\frac{u_{i}^{n+1}-u_{i}^{n}}{\delta t}+\mathscr{F} \delta x\left(u^{n}\right)_{i}+\mathscr{L}_{\alpha, \delta x}\left[u^{n+1}\right]_{i}=0 \quad \text { for all } n \geq 0 \text { and all } i \in \mathbb{Z} .
\end{gathered}
$$

where $\mathscr{F}_{\delta x}$ is any monotone consistent and consevative discretization of $\partial_{x}(f(u))$ (see e.g $[17,18,19]$ ), and $\mathscr{L}_{\alpha, \delta x}$ is a monotone discretisation of $\mathscr{L}_{\alpha}$ to be defined. Note that the scheme has explicit convection and implicit diffusion terms.

The first and simplest idea to obtain a monotone discretization of $\mathscr{L}_{\alpha}$ for $\alpha \in$ $(0,2)$ is to discretize the integral in (3) using a simple (weighted) midpoint type quadrature rule, see e.g. $[13,10,8]$. For $\varphi \in C_{c}^{\infty}(\mathbb{R})$ and letting $\varphi_{l}=\varphi\left(x_{l}\right)$ if $l \in \mathbb{Z}$, this leads to

$$
\mathscr{L}_{\alpha}[\varphi]\left(x_{i}\right) \approx \tilde{\mathscr{L}}_{\alpha, \delta x}[\varphi]_{i}:=-\sum_{j \in \mathbb{Z} \backslash\{0\}}\left(\varphi_{i+j}-\varphi_{i}\right) \int_{\left(j \delta x-\frac{\delta x}{2}, j \delta x+\frac{\delta x}{2}\right)} \frac{c_{\alpha}}{|z|^{1+\alpha}} d z
$$


However, as $\alpha \rightarrow 2$ we have $c_{\alpha} \rightarrow 0$ and therefore $\tilde{\mathscr{L}}_{\alpha, \delta x} \rightarrow 0$ for fixed $\delta x$. In the limit $\alpha \rightarrow 2$ the scheme then converges to

$$
\frac{u_{i}^{n+1}-u_{i}^{n}}{\delta t}+\mathscr{F}_{\delta x}\left(u^{n}\right)_{i}=0 \quad \text { for all } n \geq 0 \text { and all } i \in \mathbb{Z}
$$

which is a discretisation of $\partial_{t} u+\partial_{x}(f(u))=0$ and not $\partial_{t} u+\partial_{x}(f(u))-\Delta u=0$. Hence the limits $\alpha \rightarrow 2$ and $\delta x \rightarrow 0$ do not commute and the scheme is not asymptotic-preserving.

Note that $\tilde{\mathscr{L}}_{\alpha, \delta x}$ vanishes in the limit because the measure $\frac{c_{\alpha} d z}{|z|^{1+\alpha}}$ concentrates around 0 as $\alpha \rightarrow 2$, while in the above midpoint rule the integral in (3) over $\left(-\frac{\delta x}{2}, \frac{\delta x}{2}\right)$ will always be zero by symmetry. We therefore need to replace the midpoint rule on this interval by a more accurate rule based on the second order interpolation polynomial $P_{i}$ of $\varphi$ around the node $x_{i}$. We find that this polynomial satisfies $P_{i}\left(x_{i}+z\right)-P_{i}\left(x_{i}\right)-P_{i}^{\prime}\left(x_{i}\right) z=\frac{1}{2 \delta x^{2}}\left(z^{2} \varphi_{i-1}-2 z^{2} \varphi_{i}+z^{2} \varphi_{i+1}\right)$ and the new discretization therefore becomes

$$
\begin{aligned}
\hat{\mathscr{L}}_{\alpha, \delta x}[\varphi]_{i} & :=-c_{\alpha} \int_{-\frac{\delta x}{2}}^{\frac{\delta x}{2}} \frac{P\left(x_{i}+z\right)-P\left(x_{i}\right)-P^{\prime}\left(x_{i}\right) z}{|z|^{1+\alpha}} d z+\tilde{\mathscr{L}}_{\alpha, \delta x}[\varphi]_{i} \\
& =\frac{\varphi_{i+1}-2 \varphi_{i}+\varphi_{i-1}}{\delta x^{2}} \int_{\left(-\frac{\delta x}{2}, \frac{\delta x}{2}\right)} \frac{c_{\alpha}|z|^{1-\alpha}}{2} d z+\tilde{\mathscr{L}}_{\alpha, \delta x}[\varphi]_{i}
\end{aligned}
$$

We can check that the new approximation has the following truncation error [16]:

$$
\begin{aligned}
& \left|\mathscr{L}_{\alpha}[\varphi]\left(x_{i}\right)-\hat{\mathscr{L}}_{\alpha, \delta x}[\varphi]_{i}\right| \\
& \quad \leq C\left(\left\|\varphi^{(4)}\right\|_{L^{\infty}} \delta x^{4-\alpha}+\left\|\varphi^{\prime \prime}\right\|_{L^{\infty}} c_{\alpha}\left(\frac{1}{\alpha}+\frac{1}{1-\alpha}\right) \delta x^{\min (1,2-\alpha)}+\left\|\varphi^{\prime}\right\|_{L^{\infty}} \delta x\right),
\end{aligned}
$$

which is $O\left(\delta x^{2}\right)+o(1)$ as $\alpha \rightarrow 2$ and therefore does not deteriorate in this limit. Note that if $\alpha=1$, then $\frac{1}{|1-\alpha|} \delta x^{\min (1,2-\alpha)}$ must be replaced with $\delta x|\ln (\delta x)|$.

In order to obtain an approximation which uses only a finite number of discrete values, we also truncate the sum in (6) as in [13] at some index $J_{\delta x}>0$ (which may depend upon $\alpha$ ) where $J_{\delta x} \delta x \rightarrow \infty$ as $\delta x \rightarrow 0$. The final approximate operator $\mathscr{L}_{\alpha, \delta x}$ is therefore

$$
\begin{array}{r}
\mathscr{L}_{\alpha, \delta x}[\varphi]_{i}=-\sum_{0<|j| \leq J_{\delta x}} W_{\alpha, \delta x}^{j}\left(\varphi_{i+j}-\varphi_{i}\right)-W_{\alpha, \delta x}^{J_{\delta x}+1}\left(\varphi_{i-J_{\delta x}-1}-\varphi_{i}\right) \\
-W_{\alpha, \delta x}^{J_{\delta x}+1}\left(\varphi_{i+J_{\delta x}+1}-\varphi_{i}\right)-W_{\alpha, \delta x}^{0} \frac{\varphi_{i+1}-2 \varphi_{i}+\varphi_{i-1}}{\delta x^{2}}
\end{array}
$$

with weights 


$$
\begin{aligned}
& W_{\alpha, \delta x}^{0}=\int_{\left(-\frac{\delta x}{2}, \frac{\delta x}{2}\right)} \frac{c_{\alpha}|z|^{1-\alpha}}{2} d z \\
& W_{\alpha, \delta x}^{j}=\int_{\left(j \delta x-\frac{\delta x}{2}, j \delta x+\frac{\delta x}{2}\right)} \frac{c_{\alpha}}{|z|^{1+\alpha}} d z \quad \text { for } \quad 0<|j| \leq J_{\delta x}, \\
& W_{\alpha, \delta x}^{J_{\delta x}+1}=\int_{z>J_{\delta x} \delta x+\frac{\delta x}{2}} \frac{c_{\alpha}}{|z|^{1+\alpha}} d z=\int_{z<-J_{\delta x} \delta x-\frac{\delta x}{2}} \frac{c_{\alpha}}{|z|^{1+\alpha}} d z .
\end{aligned}
$$

The last term in (7) contains the classical discretization of $\varphi^{\prime \prime}\left(x_{i}\right)$ and is the new correction term compared with the discretisations of $[13,10,8]$. This discretisations fit with the generic framework of [13] from which we can conclude:

Theorem 1 ([16]). Under a standard CFL condition for the convection term,

1. There is a unique solution $u_{\alpha, \delta x, \delta t}$ of the scheme defined by (4), (5), (7) and (8), satisfying $\left\|u_{\alpha, \delta x, \delta t}\right\|_{L^{\infty}} \leq\left\|u_{i n i}\right\|_{L^{\infty}}$ and $\left|u_{\alpha, \delta x, \delta t}(t, \cdot)\right|_{B V} \leq\left|u_{\text {ini }}\right|_{B V}$ for all $t>0$.

2. For fixed $\alpha, u_{\alpha, \delta x, \delta t}$ converges in $C\left([0, \infty) ; L_{l o c}^{1}\right)$ as $(\delta x, \delta t) \rightarrow 0$ to the unique entropy solution $u_{\alpha}$ of (1).

Remark 1. We set $\mathscr{L}_{2, \delta x}[\varphi]_{i}=-\left(\varphi_{i+1}-2 \varphi_{i}+\varphi_{i-1}\right) / \delta x^{2}$ and $\mathscr{L}_{0, \delta x}[\varphi]_{i}=\varphi_{i}$. This consists in fixing $\delta x$ and sending $\alpha \rightarrow 2$ or $\alpha \rightarrow 0$ in (7). Taking the limits in the scheme (5), we obtain the classical implicit scheme for the (1) with $\alpha=2$ or $\alpha=0$.

\section{The asymptotic-preserving property}

The scheme is asymptotic-preserving if its solution $u_{\alpha, \delta x, \delta t}$ satisfies the following uniform approximation result away from $\alpha=0$ (see [16] for the case $\alpha=0$ ):

$$
\forall \eta>0, \sup _{\alpha \in[\eta, 2]} d_{L_{\mathrm{loc}}^{1}([0, \infty) \times \mathbb{R})}\left(u_{\alpha, \delta x, \delta t}, u_{\alpha}\right) \rightarrow 0 \text { as }(\delta x, \delta t) \rightarrow 0
$$

where $d_{L_{\mathrm{loc}}^{1}([0, \infty) \times \mathbb{R})}(u, v)=\sum_{n=1}^{\infty} 2^{-n} \min \left(1,\|u-v\|_{L^{1}([0, n) \times(-n, n))}\right)$ is the usual distance defining the topology of $L_{\text {loc }}^{1}([0, \infty) \times \mathbb{R})$. Here and elsewhere, the convergence $(\delta x, \delta t) \rightarrow 0$ is always taken under a standard CFL condition depending on the definition of the convective flux $\mathscr{F}$ in (5) (see e.g. [13, 10, 8]). That this formulation of the asymptotic-preserving property is very general and does not require an explicit error estimate independent on $\alpha$. Such an estimate seems particularly challenging to obtain in the absence of regularity of the solution as $t \rightarrow 0$.

Theorem 2 ([16]). Under a standard CFL for the convection part, the numerical scheme defined by (4) (5), (7) and (8) is asymptotic-preserving.

Next we want to illustrate this property numerically. As it is formulated now, this would require us to have access to the exact solution $u_{\alpha}$, which is not the case. We overcome this difficulty by using instead the following equivalent reformulation of (9) (see [16]), which can be checked by computing approximate solutions only: 
$\forall \alpha_{0} \in(0,2]$, for any sequence $\left(\delta x_{k}, \delta t_{k}\right)_{k \in \mathbb{N}}$ converging to 0 :

$$
\sup _{k \geq 1} d_{L_{\mathrm{loc}}^{1}([0, \infty) \times \mathbb{R})}\left(u_{\alpha, \delta x_{k}, \delta t_{k}}, u_{\alpha_{0}, \delta x_{k}, \delta t_{k}}\right) \rightarrow 0 \text { as } \alpha \rightarrow \alpha_{0} .
$$

Remark 2. The matrix of $\mathscr{L}_{\alpha, \delta x}$ defined by (7) is a semi-definite Toepliz matrix as in $[13,10,8]$. Implementation of the scheme thus takes advantage of super-fast multiplication and inversion algorithms for these matrices [7, 25]. Computing several approximate solutions, as required in (10), is therefore not very expensive.

\section{Numerical results}

In all these tests, we take the Burgers flux $f(u)=\frac{u^{2}}{2}$ and $\mathscr{F}_{\delta x}$ given by a MUSCL method. The final time is $T=1$ and the spatial computational domain is $[-1,1]$. We use the same truncation parameters (in particular $J_{\delta x}$ ) as in [13, Section 4.1.2].

For each test, we choose the discretisation steps $\left(\delta x_{k}, \delta t_{k}\right)=\left(\frac{1}{2^{k} \times 50}, \frac{1}{2^{k} \times 100}\right)$ for $k=1, \ldots, 4$, which all satisfy the CFL for (5). We also select four values $\left(\alpha_{m}\right)_{m=1, \ldots, 4}=(1.8,1.9,1.99,1.999)$ which are near $\alpha_{0}=2$, the difficult case in assessing the uniformity of the convergence in (10) and the reason why we introduced the correction term in (7). We then indicate, for $m=1, \ldots, 4$, the value of

$$
E_{m}=\sup _{t \in[0,1]}\left\|u_{\alpha_{m}, \delta x, \delta t}(\cdot, t)-u_{\alpha_{0}, \delta x, \delta t}(\cdot, t)\right\|_{L^{1}([-1,1])},
$$

that is the $L^{\infty}\left(L^{1}\right)$ norm of $u_{\alpha_{m}, \delta x, \delta t}-u_{\alpha_{0}, \delta x, \delta t}$ on the computational domain. This is a stronger norm that the $L^{1}\left(L^{1}\right)$ norm used in (10). Hence, $E_{m}$ approaching 0 as $m$ increases is an even better indication that the scheme is asymptotic-preserving.

Test 1 (rarefaction): we select a Riemann initial condition, $u_{\text {ini }}=-1$ if $x<0$ and $u_{\text {ini }}=1$ if $x>0$. In this case both convection and diffusion work to smooth out the intial data. Table 1 shows the values of $\left(E_{m}\right)_{m=1, \ldots, 4}$ for both the uncorrected scheme from [13] based on (6) and our corrected scheme based on (7).

Table 1 Comparison be-

tween the uncorrected scheme of [13] and our corrected scheme, $u_{\text {ini }}=-1$ on $(-\infty, 0)$, $u_{\text {ini }}=1$ on $(0, \infty)$.

\begin{tabular}{|l|c|c|c|c|}
\hline & $E_{1}$ & $E_{2}$ & $E_{3}$ & $E_{4}$ \\
\hline Uncorrected scheme & $1.8 \mathrm{E}-1$ & $3 \mathrm{E}-1$ & $8.8 \mathrm{E}-1$ & $9.1 \mathrm{E}-1$ \\
\hline Corrected scheme & $5.1 \mathrm{E}-2$ & $2.2 \mathrm{E}-2$ & $1.7 \mathrm{E}-4$ & $1.7 \mathrm{E}-5$ \\
\hline
\end{tabular}

Test 2 (smooth shock): the initial condition is $u_{\text {ini }}(x)=1$ if $x<0$ and $u_{\text {ini }}(x)=$ -1 if $x>0$. Here the hyperbolic and non-local terms in (1) compete to maintain or diffuse the initial shock. Since $\alpha_{m}$ is near 2 however, any solution is instantly smooth, but has much larger gradients near $x=0$ than the solution in Test 1 .

Both tests confirm that the scheme defined by (4), (5), (7) and (8) is asymptoticpreserving. They also confirm that, without the order 2 correction in (7), the scheme 
Table 2 Comparison between the uncorrected scheme of [13] and our corrected scheme, $u_{\text {ini }}=1$ on $(-\infty, 0)$, $u_{\text {ini }}=-1$ on $(0, \infty)$.

\begin{tabular}{|l|c|c|c|c|}
\hline & $E_{1}$ & $E_{2}$ & $E_{3}$ & $E_{4}$ \\
\hline Uncorrected scheme & $2.1 \mathrm{E}-1$ & $3.9 \mathrm{E}-1$ & 1.3 & 1.3 \\
\hline Corrected scheme & $5.3 \mathrm{E}-2$ & $2.3 \mathrm{E}-2$ & $3.2 \mathrm{E}-4$ & $4.2 \mathrm{E}-5$ \\
\hline
\end{tabular}

deteriorates as $\alpha \rightarrow 2$ and does not provide a correct numerical solution at any reasonable resolution. This is also illustrated in Figure 1, where we plot the solutions of both schemes for $\alpha=1.99$ for the initial condition of Test 2 and $(\delta x, \delta t)=$ $\left(\frac{1}{2^{4} \times 50}, \frac{1}{2^{4} \times 100}\right)$. Even at this very high resolution, the uncorrected scheme provides an incorrect approximate solution which, as expected, is closer to the solution of $\partial_{t} u+\partial_{x}(f(u))$ than to the solution of (1).

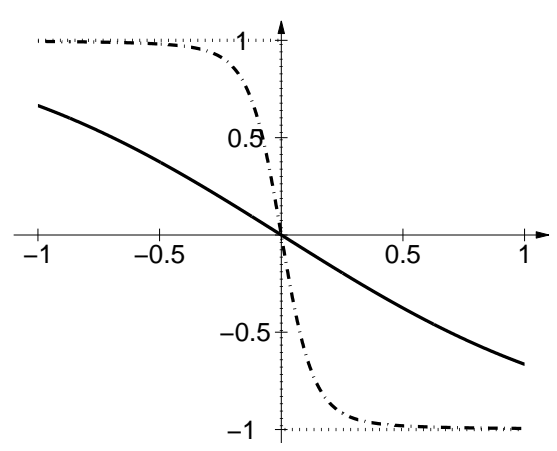

Fig. 1 Approximate solutions provided at $T=1$ by the corrected (continuous) and uncorrected (dashed) schemes for (1) with $\alpha=1.99$. The dotted line is both the initial condition and the solution to $\partial_{t} u+\partial_{x}(f(u))=0$.

\section{Conclusion}

We have presented a monotone numerical method for fractional conservation laws which is asymptotic-preserving with respect to the fractional power of the Laplacian. The scheme automatically adjusts to the change of nature of the equation as the power of the Laplacian goes to 1 (i.e. $\alpha \rightarrow 2$ in (1)) and therefore provides accurate approximate solutions for any power of the fractional Laplacian. We have given numerical results to illustrate the asymptotic-preserving property of our method, as well as the necessity of modifying previously studied monotone methods to obtain this property.

The complete theoretical study of such monotone asymptotic-preserving schemes will be presented in the forthcomming paper [16]. Here a general class of fractional degenerate parabolic equations are considered that include (1) as a special case. 


\section{References}

1. Alfaro, M., Droniou, J.: General fractal conservation laws arising from a model of detonations in gases. Appl. Math. Res. Express 2012, 127-151 (2012)

2. Alibaud, N.: Entropy formulation for fractal conservation laws. J. Evol. Equ. 7(1), 145-175 (2007)

3. Alibaud, N., Andreianov, B.: Non-uniqueness of weak solutions for the fractal Burgers equation. Ann. Inst. H. Poincaré Anal. Non Linéaire 27(4), 997-1016 (2010)

4. Alibaud, N., Droniou, J., Vovelle, J.: Occurrence and non-appearance of shocks in fractal burgers equations. J. Hyperbolic Differ. Equ. 4(3), 479-499 (2007)

5. Asmussen, S., Rosiński, J.: Approximations of small jumps of Lévy processes with a view towards simulation. J. Appl. Probab. 38(2), 482-493 (2001)

6. Biler, P., Karch, G., Woyczynski, W.: Fractal burgers equations. J. Diff. Eq. 148, 9-46 (1998)

7. Chan, R., Ng, M.: Conjugate gradient methods for toeplitz systems. SIAM Review 38(3), 427-482 (1996)

8. Cifani, S., Jakobsen, E.R.: On numerical methods and error estimates for degenerate fractional convection-diffusion equations. To appear in Numer. Math. DOI 10.1007/s00211-013-0590-0

9. Cifani, S., Jakobsen, E.R.: On the spectral vanishing viscosity method for periodic fractional conservation laws. Math. Comp. 82(283), 1489-1514 (2013)

10. Cifani, S., Jakobsen, E.R., Karlsen, K.H.: The discontinuous galerkin method for fractal conservation laws. IMA J. Numer. Anal. 31(3), 1090-1122 (2011)

11. Clavin, P.: Instabilities and nonlinear patterns of overdriven detonations in gases. Kluwer (2002)

12. Cont, R., Tankov, P.: Financial modelling with jump processes. Chapman \& Hall/CRC Financial Mathematics Series. Chapman \& Hall/CRC, Boca Raton (FL) (2004)

13. Droniou, J.: A numerical method for fractal conservation laws. Math. Comp. 79(269), 95-124 (2010)

14. Droniou, J., Gallouët, T., Vovelle, J.: Global solution and smoothing effect for a non-local regularization of an hyperbolic equation. J. Evol. Equ. 3(3), 499-521 (2003)

15. Droniou, J., Imbert, C.: Fractal first order partial differential equations. Arch. Ration. Mech. Anal. 182(2), 299-331 (2006)

16. Droniou, J., Jakobsen, E.R.: An asymptotic-preserving scheme for fractal conservation laws and frational degenerate parabolic equations. In preparation

17. Eymard, R., Gallouët, T., Herbin, R.: Finite volume methods. In: P.G. Ciarlet, J.L. Lions (eds.) Techniques of Scientific Computing, Part III, Handbook of Numerical Analysis, VII, pp. 713-1020. North-Holland, Amsterdam (2000)

18. Godlewski, E., Raviart, P.A.: Numerical approximation of hyperbolic systems of conservation laws, Applied Mathematematical Sciences, vol. 118. Springer, New-York (1996)

19. Holden, H., H., R.N.: Front tracking for Hyperbolic Conservation Laws. Springer (2002)

20. Jakobsen, E.R., Karlsen, K.H., La Chioma, C.: Error estimates for approximate solutions to Bellman equations associated with controlled jump-diffusions. Numer. Math. 110(2), 221-255 (2008)

21. Jourdain, B., Méléard, S., Woyczynski, W.: Probabilistic approximation and inviscid limits for one-dimensional fractional conservation laws. Bernoulli 11(4), 689-714 (2005)

22. Kruzhkov, S.N.: First order quasilinear equations with several independent variables. Math. Sb. (N.S.) 81(123), 228-255 (1970)

23. Soner, H.: Optimal control with state-space constraint ii. SIAM J. Control Optim. 24(6) (1986)

24. Stanescu, D., Kim, D., Woyczynski, W.: Numerical study of interacting particles approximation for integro-differential equations. Journal of Computational Physics 206, 706-726 (2005)

25. Van Loan, C.: Computational frameworks for the fast Fourier transform, Frontiers in Applied Mathematics, vol. 10. Society for Industrial and Applied Mathematics (SIAM), Philadelphia, PA (1992)

26. Woyczynski, W.: Lévy processes in the physical sciences. Birkhäuser, Boston (2001) 